\section{Entschließung der 31. Konferenz vom 15. Juni 2016}

Mit einer gemeinsamen Entschließung ${ }^{1}$ vom 15.06.2016 ruft die Konferenz der Informationsfreiheitsbeauftragten in Deutschland alle Bundesländer zur Teilnahme an dem Datenportal „GovData“ auf.

Transparenz und Akzeptanz des Verwaltungshandelns sowie nicht zuletzt das immense wirtschaftliche Potential: Die Vorteile von Open Data liegen auf der Hand. Open Data bezeichnet die zentrale Verfügbarkeit und bessere Nutzbarkeit von Verwaltungsinformationen. „GovData - das Datenportal für Deutschland“ bietet einen einheitlichen zentralen Zugang zu offenen Verwaltungsinformationen aus Bund, einigen Kommunen und noch nicht allen Bundesländern. Mit einer gemeinsamen Entschließung vom 15.06.2016 appelliert die Konferenz der Informationsfreiheitsbeauftragten in Deutschland an alle Bundesländer, sich an dem Datenportal zu beteiligen.

Helga Block, Landesbeauftragte für Datenschutz und Informationsfreiheit NordrheinWestfalen und diesjährige Vorsitzende der Konferenz der Informationsfreiheitsbeauftragten in Deutschland: „GovData als zentrales Datenportal mit Suchfunktion trägt zur besseren Nutzbarkeit von amtlichen Informationen bei. Damit ist es sowohl ein wichtiger Bestandteil von Open Data als auch ein weiterer Beitrag zu mehr Transparenz."

\section{GovData: Alle Länder sollen der Verwaltungsvereinbarung beitreten und Daten auf dem Portal bereitstellen!}

„GovData - das Datenportal für Deutschland“ ist eine Anwendung des IT-Planungsrats, die auf der Grundlage einer Verwaltungsvereinbarung vom Bund und mehreren Ländern betrieben wird. Das Portal bietet einen einheitlichen zentralen Zugang zu offenen Verwaltungsdaten aus Bund, Ländern und Kommunen. Ziel ist es, diese Daten möglichst flächendeckend zur Verfügung zu stellen und sie an einer zentralen Stelle auffindbar und so einfacher nutzbar zu machen. GovData dient damit nicht nur der Information der Bürgerinnen und Bürger, sondern fördert zugleich auch die Transparenz und Akzeptanz des Verwaltungshandelns. Es stellt der Wirtschaft darüber hinaus Verwaltungsdaten zur Entwicklung neuer Geschäftsmodelle zur Verfügung.

Bislang beteiligen sich jedoch an dem Bund-Länder-Online-Portal noch nicht alle Länder. Viele Daten, an deren Veröffentlichung ein großes öffentliches Interesse besteht, sind noch nicht abrufbar. Das immense wirtschaftliche Potential von Open Data bleibt ungenutzt.

Sowohl für die Wirtschaft als auch für die Zivilgesellschaft ergeben sich erhebliche Vorteile durch einen freien Zugang zu den öffentlichen Daten der Verwaltung. Der Umfang und die Qualität der in GovData zur Verfügung gestellten Daten müssen verbessert und der Nutzwert des Portals weiter erhöht werden.

Daher appelliert die Konferenz der Informationsfreiheitsbeauftragten in Deutschland an die verbleibenden Länder, der Verwaltungsvereinbarung beizutreten, und fordert alle Vereinbarungspartner zur verstärkten Bereitstellung von Daten auf.

\section{Springer Vieweg}
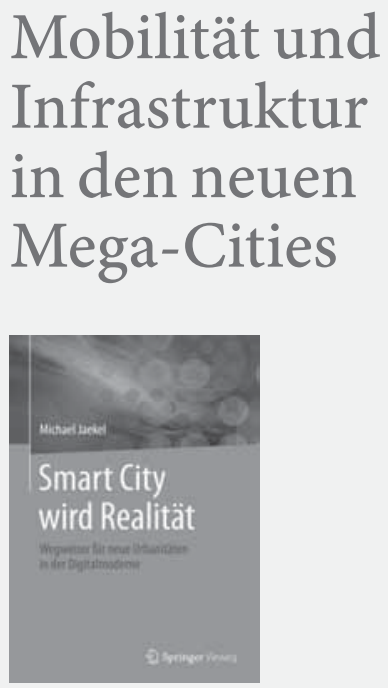

Michael Jaekel

Smart City wird Realität

1. Aufl. 2015. XVI, 312 S.

$108 \mathrm{Abb}$. Brosch.

$€(D) 49,99|€(A) 51,39|{ }^{*} \operatorname{sFr} 53,00$

ISBN 978-3-658-04454-1 (Print)

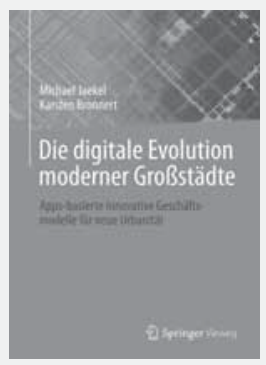

Michael Jaekel; Karsten Bronnert Die digitale Evolution moderner Großstädte

2013. X, 190 S. 51 Abb. Brosch. $€$ (D) $52,99 \mid €$ (A) $54,47 \mid{ }^{*} \mathrm{sFr} 56,00$ ISBN 978-3-658-00170-4 (Print)

$€(D)$ sind gebundene Ladenpreise in Deutschland und enthalten $7 \%$ MwSt. $€$ (A) sind gebundene Ladenpreise in Österreich und enthalten $10 \%$ MwSt. Die mit * gekennzeichneten Preise sind unverbindliche Preisempfehlungen und enthalten die landesübliche MwSt. Preisänderungen und Irrtümer vorbehalten.

springer-vieweg.de 